\title{
Transmission Electron Microscopy Study of Iron Oxide Poisoning in Fluid Catalytic Cracking Catalysts
}

\author{
Y. L. Tang, ${ }^{*}$ D.A. Cullen, ${ }^{* *}$ D.W. Coffey, ${ }^{* *}$ M. Allahverdi, ${ }^{*}$ W.J. Reagan* and L.F. Allard** \\ *Johnson Matthey Intercat Technology Center, Savannah, GA 31408 \\ **Materials Science \& Technology Div., Oak Ridge National Laboratory, Oak Ridge, TN 37831
}

Iron contamination of fluid catalytic cracking (FCC) catalysts has become a serious and expensive problem for the oil refinery industry. Iron from feed oil deposits on the outer surface of the catalyst particles, leading to reduced activity and bottom conversion efficiency. In addition, catalyst circulation problems often occur due to changes in the bulk density of the equilibrium catalyst. This problem is more severe when refiners choose to run the more plentiful residual feedstock or other opportunity crude oils with high iron content. Efforts to develop high iron tolerance FCC catalysts and/or additives have, as of yet, been largely unsuccessful. An understanding of the phase structure, especially the interface between the iron compounds and catalyst components, is a key to finding a method to control the iron poisoning phenomenon.

FCC catalyst surfaces were prepared in cross-section for TEM analysis by focused-ion-beam (FIB) milling. A site-specific lamina was prepared at the surfaces of two closely neighboring microspheres supported in an epoxy matrix, as shown in Figure 1. The specimen was carefully thinned to electron transparency, using a final ion-beam energy of $5 \mathrm{keV}$. A probe-corrected JEOL 2200FS scanning transmission electron microscope (STEM), equipped with a Bruker X-Flash Si drift detector (SDD) for energy dispersive spectroscopy (EDS), was used to elucidate the phase constituents in the surface reaction layers. EDS maps clearly show an Fe phase on the surfaces of both particles (FIG. 2). X-ray diffraction data suggest that this surface layer consists of randomly oriented $\mathrm{Fe}_{2} \mathrm{O}_{3}$ and $\mathrm{F}_{3} \mathrm{O}_{4}$ crystalline phases distributed discontinuously on the surface. The sizes of the crystalline phase are about $20 \sim 100 \mathrm{~nm}$. EDS and selected area electron diffraction (SAED) analysis both confirmed the existence of the iron oxides (FIG. 3).

Underneath the crystalline iron oxides were thicker amorphous layers which are the main component of the "iron ring" which has been observed by analytical scanning electron microscopy [1,2]. Zcontrast imaging showed this amorphous region had a layered structure (FIG. 3). This new founding suggests that the layers formed through deposition of an iron rich phase in the FCC unit over a period of time. It can be speculated that the thickness of this amorphous layer is proportional to the amount of time the catalyst spent in contact with the iron rich feed.

\section{References}

[1] George Yaluris et al., NPRA Annual Meeting 2001, AM-01-59.

[2] L.T. Boock, et al., ACS Symposium Series 634 (1996) 171.

[3] This research is partly supported by the SHaRE User Facility, which is an Office of Science User Facility operated for the U.S. Department of Energy (DOE) Office of Science by the Oak Ridge National Laboratory (ORNL). ORNL is sponsored by the DOE, Office of Energy Efficiency and Renewable Energy, Industrial Technologies Program, under contract DE-AC05-00OR22725 with UT-Battelle, LLC. 

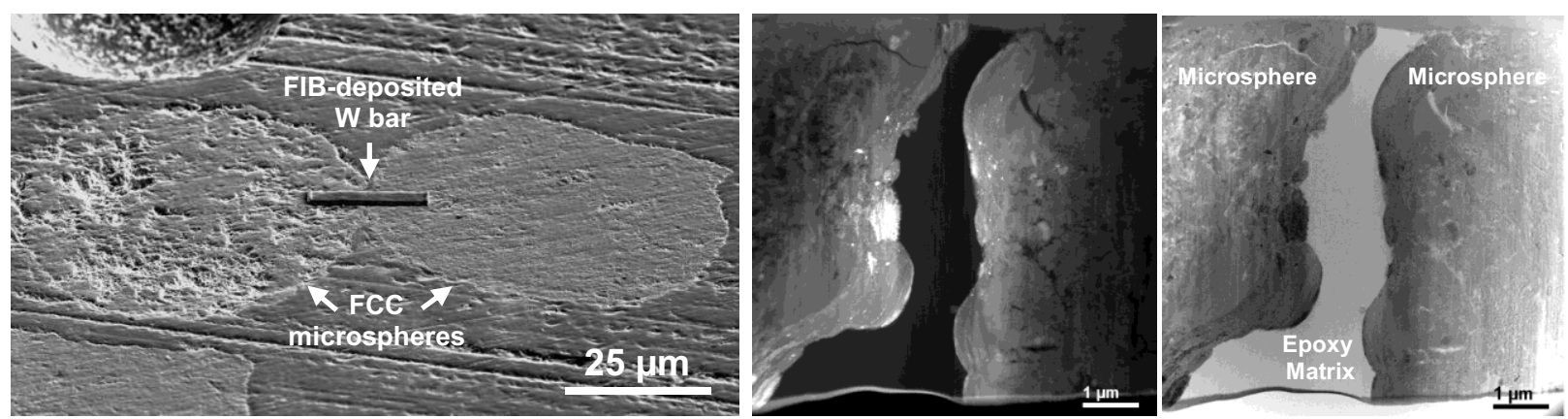

FIG. 1. (left) SEM image of two neighboring FCC microspheres. A FIB x-section was prepared across the surfaces of the two spheres, at the location marked by the $\mathrm{W}$ protection layer. (middle) STEM dark-field (DF) and (right) bright-field (BF) images of the x-section specimen.
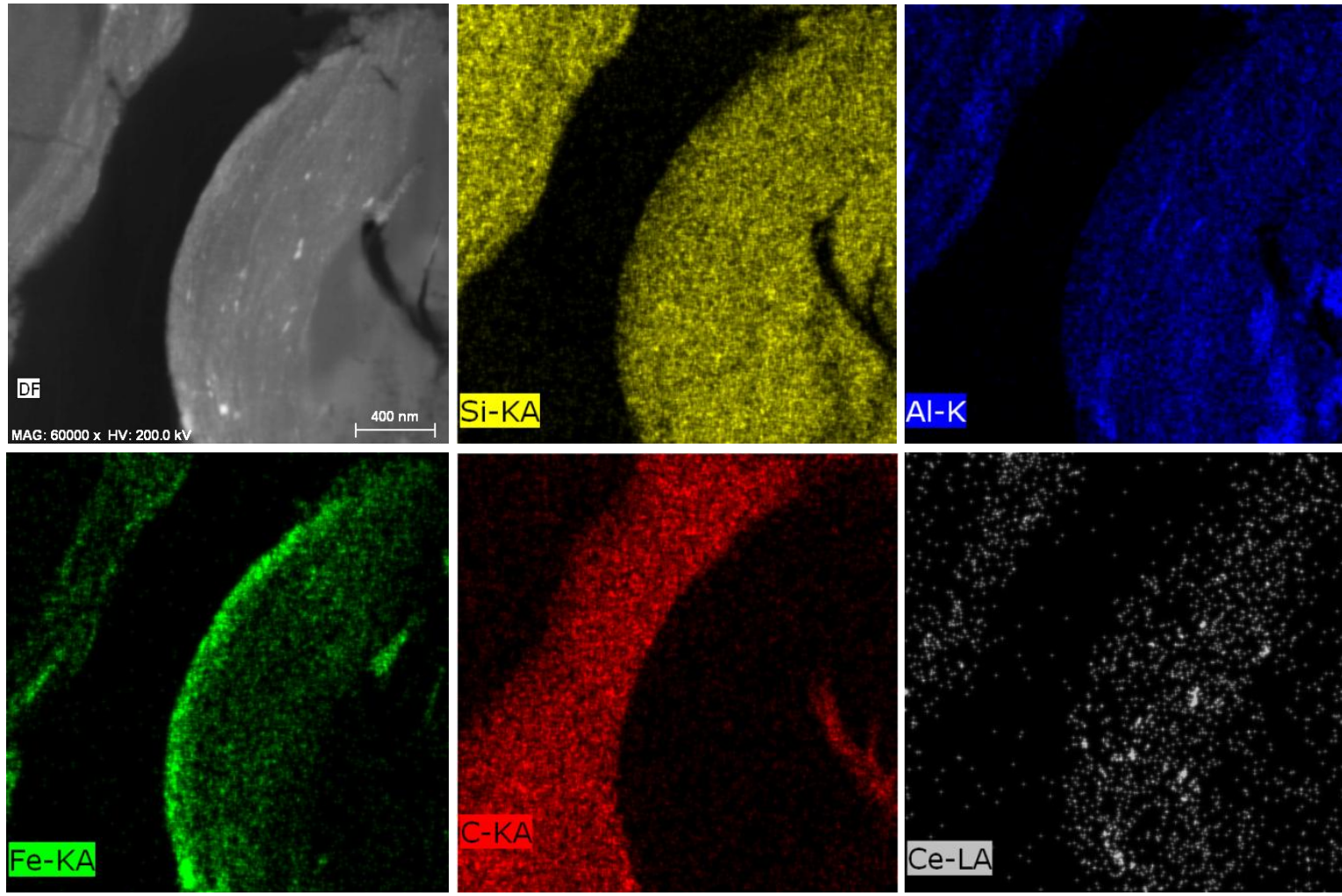

FIG. 2. STEM Z-contrast image and EDS maps of the surface structure of two FCC catalysts. The outermost shell consisted of crystalline iron oxide, which transitioned into an iron rich amorphous phase deeper into the microsphere.

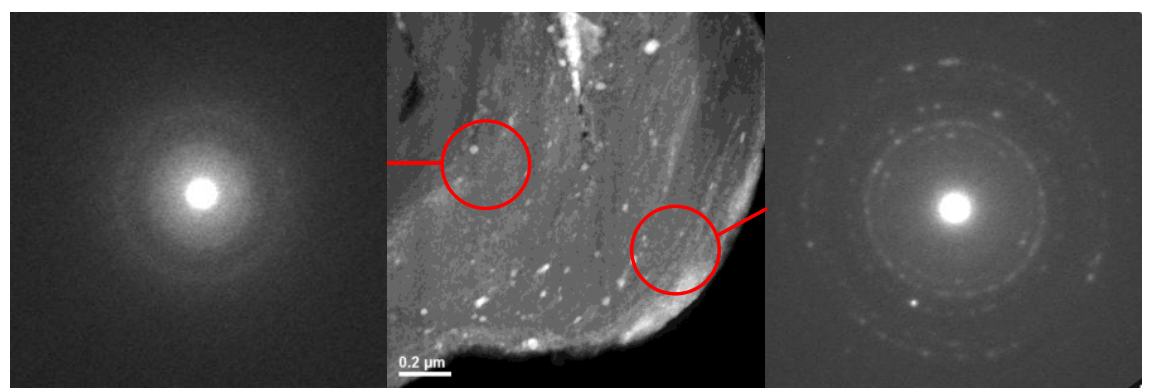

FIG. 3. Z-contrast image and SAED patterns showing the crystalline iron oxide phase on the microsphere surface and the amorphous phase within the matrix. 